\title{
RELAÇÃO ENTRE EMOÇÕES E AUTOCONTROLE EM CRIANÇAS E ADOLESCENTES
}

\author{
Relation Between Emotions and Self-control \\ in Children and Adolescents
}

\author{
Fabián Javier Marín Rueda ${ }^{1}$ \\ Fermino Fernandes Sisto ${ }^{2}$
}

\section{Resumo}

O objetivo da pesquisa foi verificar possíveis relações entre as emoções e o autocontrole. Para isso, aventaram-se duas hipóteses, quais sejam, um autocontrole elevado estaria associado a um controle das emoções negativas, enquanto um baixo autocontrole estaria associado às emoções positivas. Ainda, pensou-se na possibilidade do autocontrole relacionado a sentimentos e emoções apresentar magnitudes de correlação maiores que o autocontrole de normas e condutas sociais. Participaram 1232 crianças e adolescentes de ambos os sexos, com idade variando de 8 a 14 anos, de cidades do interior do estado de São Paulo. Os instrumentos utilizados foram a Escala de Autopercepção de Emoções e Escalas Feminina e Masculina de Autocontrole, tendo ocorrido a aplicação de forma coletiva em sala de aula. Os resultados evidenciaram, de forma geral, correlações de magnitudes consideradas nulas ou baixas entre as diferentes emoções e o autocontrole de normas e condutas sociais. As emoções tristeza e medo apresentaram correlações de magnitude moderada com o fator do autocontrole referente a sentimentos e emoções, o que pode ser considerado uma evidência de validade concorrente.

Palavras-chave: Emoções; Autocontrole; Validade.

Psicólogo, Mestre em Avaliação Psicológica e Doutorando do Programa de Pós-Graduação Stricto Sensu em Psicologia da Universidade São Francisco. Bolsista CAPES. São Paulo - SP. e-mail: marinfabian@yahoo.com.br e-mail: fermino.sisto@gmail.com

2 Doutor pela Universidad Complutense de Madrid, Livre-docente pela Unicamp e docente do Programa de Pós-Graduação Stricto Sensu em Psicologia da Universidade São Francisco. Campinas - SP. 


\section{Abstract}

The objective of this study was to verify possible relations between emotions and self-control, considering two hypotheses. The first one is that a high self-control would be associated with a control of the negative emotions, while a low self-control would be associated with the positive emotions. The second is the possibility of self-control of norms and social behaviors to present inferior magnitude of correlation if compared with the self-control related to the feelings and emotions Participated of the research 1232 children and adolescents of both sex, with ages between 8 and 14 years old, from the state of São Paulo. The Escala de Autopercepção de Emoções and Escalas Feminina e Masculina de Autocontrole were the instruments used and applied of collective form in the classroom. The results evidenced null or low correlations of magnitudes between different emotions and self-control of norms and social behaviors. Emotions like sadness and fear presented moderate correlations of magnitude with the self-control factor regarding feelings and emotions, which can be considered as validity evidence.

Keywords: Emotions; Self-control; Validity.

\section{Introdução}

As emoções possuem seu núcleo na experiência de prazer ou dor, inserido na consciência da estrutura de significados de uma dada situação. Dessa forma, a percepção da pessoa em relação à situação e o significado emocional proveniente dessa percepção fundamentam as emoções e sua intensidade (Frijda, 1995). Nesse contexto, pode-se dizer que as emoções tratam de respostas às estruturas de significados de determinadas situações e, em decorrência, diferentes estruturas poderiam favorecer o aparecimento de distintas emoções.

Além disso, as emoções não estariam apenas relacionadas às condições favoráveis ou desfavoráveis, mas também às suas mudanças reais ou imaginárias. Assim, quanto mais radical uma mudança, mais intensas serão as emoções. Contudo, as mudanças emocionais não se dão da mesma forma para as emoções positivas e negativas. Isso porque o prazer é contingente à mudança e desaparece na presença da satisfação contínua, mas a dor não desaparece se condições adversas persistirem (Sisto, Bartholomeu, Rueda \& Fernandes, 2004).

Deve ser destacado que um conceito importante no estudo das emoções são as chamadas emoções básicas ou primárias, como alegria, tristeza, medo e coragem (Frijda, 1995). No Brasil, elas podem ser avaliadas pelo instrumento de Autopercepção de Emoções (Sisto \& Bazi, 1999).

Sisto e Bazi (1999) consideram a alegria como uma forma de prazer relacionada à euforia e à busca de contato e concordam com Misrahi (2001) de que seja uma atividade da consciência, pois envolve seus significados e valores. Defendem, como Dolores Avia (1995), que a alegria estimula a flexibilidade cognitiva e respostas inovadoras, sendo que a busca de estímulos pode ser a fonte de motivação intrínseca.

Já a tristeza foi definida como uma forma de desprazer aliada à retirada de desejo e sensação de estar indefeso, conforme proposto por Frijda (1995). Além disso, a tristeza também possibilita o embotamento afetivo, a imobilidade e lentidão fisiológica, em consonância ao proposto por Marino Júnior (1975). Enquanto a alegria está associada à formação de vínculos sociais, a tristeza reduz a atividade da pessoa, que busca restaurar sua energia por meio da simpatia e atenção alheias. De acordo com Verztman (1995), a tristeza é caracterizada como uma fase passageira, diluída pela vida cotidiana.

O medo foi considerado como uma emoção que paralisa o indivíduo e relaciona-se a atitudes evitadoras e autoprotetoras. Envolve o desenvolvimento de formas de comportamentos, estímulos sinalizadores produzidos durante esses comportamentos e mecanismos de reconhecimento de tais estímulos. Destaca-se que o medo se diferencia da ansiedade por haver uma ameaça concreta presente, o que não ocorre na ansiedade, uma vez que ela aparece devido à antecipação de uma ameaça futura (Frijda, 1995).

Finalmente, a emoção coragem é a capacidade de "seguir em frente", apesar do desespero (May, 1975). A coragem possui como uma das principais características o sacrifício de peculiaridades do indivíduo, sem o qual não seria possível realizar o ato de coragem. 
Por sua vez, o autocontrole pode ser definido como uma forma de optar por certos comportamentos, geralmente em situações conflituosas, de acordo com padrões definidos pela sociedade (Martinelli \& Sisto, 2007). É uma variável estudada a partir de muitas perspectivas, sendo que cada uma delas foca aspectos específicos desse tipo de comportamento.

Embora o autocontrole tenha sido pesquisado em vários contextos, como, por exemplo, na área organizacional (Konopkin, Stepanskii \& Kondrat'eva, 1973; Herman, 1971, dentre outros), e na área clínica (Gold \& Cundiff, 1980; Harris \& Johnson, 1980, por exemplo), a maior quantidade de estudos é encontrada na área da educação (Dutrow \& Houston, 1981; Kearney, 1966; Saltz \& Meade, 1973; Wolfe \& Johnson, 1995, entre outros).

$\mathrm{Na}$ educação, estudos têm apontado o autocontrole como um bom previsor do desempenho acadêmico (Agostín \& Bain, 1997; Kearney, 1966; Whiteside, 1966, por exemplo). Ao lado disso, autores como Harris e Graham (1985), Sappington, Fritschi, Sandefer e Tauxe (1980) e Stevenson e Fantuzzo (1986) também sugerem que o treinamento do autocontrole poderia conduzir a uma melhoria no rendimento escolar.

As Escalas Feminina e Masculina de Autocontrole (EFA \& EMAC respectivamente) têm como objetivo avaliar de forma precisa dois diferentes tipos de autocontrole (em relação às regras e condutas sociais e em relação a sentimentos e emoções) de crianças e jovens, valendo-se de situações com as quais eles interagem freqüentemente.

O estudo realizado por Martinelli e Sisto (2007) sobre evidência de validade pelo funcionamento diferencial do item (DIF) indicou muitos itens que diferenciaram os sexos, em razão do qual os autores construíram duas escalas, uma para cada sexo, sendo cada escala composta por itens comuns a ambos os sexos e itens característicos do respectivo sexo. Dessa forma, a escala feminina ficou composta por 30 itens e a escala masculina por 31 itens.

Para interpretar cada fator, foi levada em consideração a nucleação das frases, com base no conteúdo semântico. Em ambas as escalas, os fatores foram interpretados como autocontrole em relação a regras e condutas sociais (fator 1 na escala masculina e fator 2 na feminina) e autocontrole em relação a sentimentos e emoções (fator 2 na escala masculina e fator 1 na feminina).
Quanto às propriedades psicométricas, as evidências de validade da estrutura interna dos itens, tanto da escala feminina como da masculina, e a correlação item-total mostraram-se dentro dos parâmetros esperados. Quanto à validade de construto em relação ao desenvolvimento, as análises de ambas as escalas sugeriram um possível caráter evolutivo do autocontrole.

Em relação à precisão das escalas, ela foi calculada pelo alfa de Cronbach e pelo método de teste-reteste. A consistência interna, avaliada pelo alfa, forneceu índices entre 0,73 e 0,84 , considerados muito bons. No caso do teste-reteste, em ambas as escalas, os autores concluíram que os fatores possuem uma estabilidade temporal bastante boa, com índices variando entre 0,73 e 0,86.

No caso das evidências quanto a critérios externos, Sisto e Rueda (2006) estudaram sua relação com os traços de personalidade avaliados pela Escala de Traços de Personalidade para crianças (Sisto, 2004). Os resultados evidenciaram a existência de relação entre ambos os construtos tanto nos meninos quanto nas meninas, fornecendo dessa forma mais uma evidência de validade para as Escalas Feminina e Masculina de Autocontrole.

Destaca-se que embora as escalas de autocontrole apresentem evidências de validade quanto à estrutura interna e bons índices de precisão, assim como evidência de validade em relação aos traços de personalidade, nenhum estudo se deteve na análise das relações que os diferentes tipos de autocontrole estabelecem com as diferentes emoções. Entretanto, é esperado que, considerando as características das emoções e que um dos fatores se refere ao autocontrole de sentimentos e emoções, elas apresentem relações com variáveis como o autocontrole. É nesse contexto que este estudo se insere, procurando contribuir para a implementação desses instrumentos e maior compreensão dos dois construtos.

Nesse sentido, partiu-se da hipótese de que pessoas com um autocontrole elevado apresentariam um controle também das emoções negativas. Em relação às emoções positivas isso não seria observado, pois o fato de elas serem consideradas positivas ou socialmente aceitas e "bem vistas" não faria com que os indivíduos necessitassem controlá-las. Ao lado disso, espera-se que as correlações com o fator de autocontrole que se refere a sentimentos e emoções sejam em maior quantidade e magnitudes do que com o fator referente a normas e condutas sociais. 


\section{Método}

\section{Participantes}

Participaram dessa pesquisa 1232 crianças e adolescentes, de 8 a 14 anos, com uma idade média de 10,38 anos $(D P=1,79)$. Com relação ao sexo, 49,3\% eram meninos e $50,7 \%$ meninas, todos provenientes de escolas públicas e particulares de uma cidade do interior de São Paulo. De forma geral, a maior freqüência de crianças por idade esteve de acordo com a série que, de fato, deveriam estar freqüentando. Houve ainda uma menor concentração de pessoas na oitava série (22 participantes) em detrimento das demais séries. Para uma melhor visualização das idades, série e sexo dos participantes da pesquisa, é apresentada a Tabela 1.

\section{Tabela 1 - Freqüência por idade, sexo e série das crianças participantes da pesquisa}

\begin{tabular}{|c|c|c|c|c|c|c|c|c|c|}
\hline \multirow[t]{2}{*}{ Série } & \multirow[t]{2}{*}{ Sexo } & \multicolumn{7}{|c|}{ Idade } & \multirow[t]{2}{*}{ Total } \\
\hline & & 8 & 9 & 10 & 11 & 12 & 13 & 14 & \\
\hline \multirow[t]{2}{*}{$2^{\mathrm{a}}$} & Masculino & 83 & 37 & 4 & 2 & 1 & 0 & 0 & 127 \\
\hline & Feminino & 94 & 41 & 3 & 1 & 0 & 0 & 0 & 139 \\
\hline \multirow[t]{2}{*}{$3^{a}$} & Masculino & 3 & 108 & 57 & 11 & 3 & 1 & 1 & 184 \\
\hline & Feminino & 1 & 108 & 36 & 4 & 3 & 0 & 0 & 152 \\
\hline \multirow[t]{2}{*}{$4^{a}$} & Masculino & 0 & 3 & 64 & 42 & 22 & 5 & 0 & 136 \\
\hline & Feminino & 0 & 2 & 85 & 51 & 8 & 5 & 1 & 152 \\
\hline \multirow[t]{2}{*}{$5^{a}$} & Masculino & 0 & 0 & 1 & 18 & 18 & 15 & 8 & 60 \\
\hline & Feminino & 0 & 0 & 0 & 28 & 20 & 11 & 4 & 63 \\
\hline \multirow[t]{2}{*}{$6^{a}$} & Masculino & 0 & 0 & 0 & 1 & 30 & 16 & 9 & 56 \\
\hline & Feminino & 0 & 0 & 0 & 0 & 34 & 25 & 6 & 65 \\
\hline \multirow[t]{2}{*}{$7^{a}$} & Masculino & 0 & 0 & 0 & 0 & 1 & 12 & 19 & 32 \\
\hline & Feminino & 0 & 0 & 0 & 0 & 1 & 31 & 12 & 44 \\
\hline \multirow[t]{3}{*}{$8^{a}$} & Masculino & 0 & 0 & 0 & 0 & 0 & 1 & 11 & 12 \\
\hline & Feminino & 0 & 0 & 0 & 0 & 0 & 0 & 10 & 10 \\
\hline & Total & 181 & 299 & 250 & 158 & 141 & 122 & 81 & 1232 \\
\hline
\end{tabular}

Para estudar a discriminação de medidas, as pessoas com pontuações extremas nas Escalas Feminina e Masculina de Autocontrole foram selecionadas, ficando configurado um novo grupo para o estudo. Assim, nos dois grupos de pontuações extremas $(25 \%$ de crianças com menor pontuação e $25 \%$ de crianças com maior pontuação) em relação ao autocontrole, a idade média foi 9,54 com um desvio-padrão de 1,56, de um total de 597 crianças e adolescentes.

\section{Instrumentos}

\section{Escala de Autopercepção de Emoções (Sisto \& Bazi, 1999)}

O instrumento é composto por 32 itens, possuindo validade de construto e precisão para quatro fatores, quais sejam, alegria, tristeza, medo e coragem. A faixa etária de abrangência é de 8 a 14 anos de idade. Cada subescala contém 8 afirmações para as quais o sujeito deve responder sim ou não. As frases estão distribuídas ao acaso e o gabarito permite agrupá-las. Para cada resposta sim é atribuído 1 ponto e para a resposta não, 0 ponto. $\mathrm{O}$ total em cada fator é fornecido pela soma dos itens pertinentes.

\section{Escalas Feminina e Masculina de Autocontrole (Martinelli \& Sisto, 2006)}

É uma escala que se propõe avaliar dois fatores do autocontrole, quais sejam, autocontrole em relação a regras e condutas sociais (fator 1 no EMAC e fator 2 no EFAC) e autocontrole em relação a sentimentos e emoções (fator 2 no EMAC e fator 1 no EFAC), em crianças de 8 a 14 anos. Contém 30 
questões na escala feminina e 31 questões na masculina, que retratam situações que podem ser vivenciadas pelas crianças e adolescentes no seu diaa-dia, como, por exemplo, "fico muito nervoso quando tenho que falar para a classe" (autocontrole em relação a regras e condutas sociais), "fico irritado quando não sei fazer alguma coisa" (autocontrole em relação sentimentos e emoções).

É composto por uma escala Likert de três pontos (sempre, às vezes e nunca). As questões podem ser positivas ou negativas. Para as positivas, à resposta "sempre" são atribuídos 2 pontos, "às vezes", 1 ponto e "nunca", zero ponto. Para as questões negativas, "sempre" corresponde a zero ponto, "às vezes", 1 ponto e "nunca", 2 pontos.

\section{Procedimento}

Os instrumentos foram aplicados coletivamente em sala de aula, por uma equipe composta por quatro psicólogas e duas pedagogas com conhecimento prévio dos testes, sendo que em cada classe permaneciam duas aplicadoras, uma para dar as instruções e a outra para auxiliar os participantes individualmente. Para ambos os testes, cada sujeito possuía o instrumento com os itens e as alternativas para serem assinaladas. $O$ aplicador lia cada item e esperava que as crianças e os adolescentes anotassem sua resposta, fazendo um "X" na alternativa escolhida, após um breve treinamento para tal.

\section{Resultados}

Todas as análises realizadas foram para cada sexo de forma separada em razão da escala de autocontrole. Dessa forma, num primeiro momento são apresentadas as estatísticas descritivas das emoções e dos fatores de autocontrole para o sexo masculino e feminino. Posteriormente foram correlacionadas as emoções com os fatores das Escalas Feminina e Masculina de Autocontrole de forma geral, com o sem controle da idade e, por fim, os grupos extremos em relação às emoções foram comparados por meio da prova $t$ de student. As estatísticas descritivas das medidas trabalhadas na pesquisa encontram-se na Tabela 2 .

\section{Tabela 2 - Estatísticas descritivas da escala de autopercepção de emoções e da escala da percepção do autocontrole}

\begin{tabular}{lllllllll}
\hline \multirow{2}{*}{ Medidas } & \multicolumn{3}{c}{ Sexo Masculino } & \multicolumn{3}{c}{ Sexo Feminino } \\
& $M$ & $D P$ & Mínimo & Máximo & $M$ & $D P$ & Mínimo & Máximo \\
\hline Alegria & 4,51 & 0,71 & 0 & 8 & 4,48 & 0,68 & 0 & 8 \\
Tristeza & 7,11 & 0,91 & 0 & 8 & 6,76 & 1,01 & 0 & 8 \\
Medo & 6,83 & 0,95 & 0 & 8 & 6,16 & 0,96 & 0 & 8 \\
Coragem & 5,07 & 0,92 & 0 & 8 & 5,97 & 0,98 & 0 & 8 \\
RCS & 21,56 & 5,22 & 3 & 30 & 23,69 & 6,07 & 1 & 39 \\
SE & 16,30 & 5,57 & 2 & 32 & 14,38 & 3,21 & 4 & 20 \\
& & & & & & & & \\
\hline
\end{tabular}

RCS $=$ fator referente às regras e condutas sociais

$\mathrm{SE}=$ fator referente a sentimentos e emoções

Na Tabela 2 observa-se que todas as medidas relativas às emoções apresentaram as pontuações mínimas e máximas possíveis. Em relação ao autocontrole, os meninos apresentaram pontuações levemente maiores no fator RCS e as meninas tiveram maiores pontuações no fator SE. $\mathrm{Na}$ Tabela 3 estão os coeficientes de correlação de Pearson para os fatores referentes às regras e condutas sociais do EMAC e do EFAC, com e sem controle da idade. 


\section{Tabela 3 - Coeficientes de correlação de Pearson e correlações parciais entre as emoções e o fator das regras e condutas sociais do EMAC e do EFAC}

\begin{tabular}{llcll}
\hline & \multicolumn{5}{c}{ Coeficientes de correlação de Pearson } \\
& Alegria & Tristeza & Medo & Coragem \\
& $r$ & $r$ & $r$ & $r$ \\
EMAC & $-0,20$ & 0,27 & 0,02 & $-0,03$ \\
EFAC & $-0,23$ & 0,17 & $-0,03$ & 0,03 \\
& & \multicolumn{5}{c}{ Coeficientes de correlação parcial } \\
& Alegria & Tristeza & Medo & Coragem \\
& $r$ & $r$ & $r$ & $r$ \\
EMAC & $-0,21$ & 0,08 & 0,05 & $-0,03$ \\
EFAC & $-0,27$ & 0,19 & 0,04 & $-0,01$ \\
\hline
\end{tabular}

Como evidencia a Tabela 3, em relação às magnitudes das correlações, salienta-se que a tristeza e o fator do EMAC e a alegria com o EFAC e o EMAC, com e sem controle do efeito da idade, apresentaram correlações baixas, mas não nulas (de $0,20$ a 0,40$)$. Quando analisados os dados controlando o efeito da idade, verificou-se que as magnitudes mantiveram-se semelhantes, exceção feita à relação entre a tristeza e o fator do EMAC. Ou seja, das oito correlações possíveis sem o controle do efeito da idade, três apresentaram magnitudes baixas e cinco magnitudes nulas. Quando controlado o efeito da idade, esse número abaixou para duas correlações apenas, isto é, a correlação com tristeza, de baixa passou a nula. Porém, pôde-se sugerir que exista, independentemente da idade, uma parte da variância comum entre as emoções avaliadas pela Escala de
Autopercepção de Emoções e o fator referente a normas e condutas sociais do EMAC e do EFAC. Essa comunalidade sugere que conforme aumenta o nível de tristeza das crianças, aumenta também seu autocontrole em relação ao fator das regras e condutas sociais; enquanto que conforme aumenta o nível de alegria, diminui o autocontrole das crianças. Já em relação às emoções medo e coragem, todas as correlações apresentaram magnitudes consideradas nulas, o que sugere que os instrumentos não apresentam comunalidade.

Também com o objetivo de verificar a relação entre as emoções e o fator relacionado aos sentimentos e emoções do EMAC e do EFAC, foram calculados os coeficientes de correlação entre as medidas e também controlado o efeito da idade. Os resultados podem ser observados na Tabela 4.

\section{Tabela 4 - Coeficientes de correlação de Pearson e correlações parciais entre as emoções e o fator referente a sentimentos e emoções no EMAC e EFAC}

\begin{tabular}{|c|c|c|c|c|}
\hline & \multicolumn{4}{|c|}{ Coeficientes de correlação de Pearson } \\
\hline & Alegria & Tristeza & Medo & Coragem \\
\hline & $r$ & $r$ & $r$ & $r$ \\
\hline EMAC & $-0,05$ & 0,35 & 0,27 & 0,01 \\
\hline \multirow[t]{4}{*}{ EFAC } & $-0,23$ & 0,48 & 0,29 & 0,06 \\
\hline & \multicolumn{4}{|c|}{ Coeficientes de correlação parcial } \\
\hline & Alegria & Tristeza & Medo & Coragem \\
\hline & $r$ & $r$ & $r$ & $r$ \\
\hline EMAC & $-0,05$ & 0,35 & 0,27 & 0,01 \\
\hline EFAC & $-0,24$ & 0,48 & 0,31 & 0,05 \\
\hline
\end{tabular}


Diferentemente do fator referente às normas e condutas sociais, no autocontrole de sentimentos e emoções dos oito coeficientes estudados, cinco não foram nulos, sendo quatro de magnitude baixa e um moderada, que foi entre a emoção tristeza e o fator do EFAC. Nesse caso, verificou-se que quanto mais tristeza nas meninas, maior será o autocontrole das suas emoções e sentimentos.

\section{Discussão}

Este estudo foi proposto com base na possibilidade do autocontrole relacionar-se às emoções. Assim, partiu-se do pressuposto de que crianças e adolescentes com um elevado autocontrole apresentariam também uma pontuação elevada nas emoções consideradas negativas (tristeza e medo), enquanto a pontuação nas emoções positivas (alegria e coragem) tenderia a ser menor. Além disso, pensou-se na possibilidade de haver uma maior relação entre o autocontrole relacionado a sentimentos e emoções a as emoções estudadas do que com o autocontrole das normas e condutas sociais. Para discussão dos resultados, não existe a possibilidade de relacionar os achados desta pesquisa com a literatura, pois infelizmente não foram encontrados estudos relacionando os construtos pesquisados nem investigações com os instrumentos trabalhados.

No caso dos fatores do EMAC e do EFAC que se referem ao autocontrole das regras e condutas sociais, houve magnitudes de correlação baixas com as emoções alegria e tristeza, e magnitudes nulas com o medo e a coragem. Embora os coeficientes tenham sido maiores com a alegria e a tristeza, os resultados de uma forma geral podem ser considerados uma evidência de validade entre os instrumentos.

Assim, os construtos psicológicos analisados nesta pesquisa pareceriam incluir mecanismos psicológicos diferentes um do outro. Em outros termos, embora as hipóteses quanto às correlações positivas e negativas tenham sido verificadas, o fato de as crianças se autocontrolarem em relação às regras e condutas sociais aconteceria independentemente delas terem uma ou outra emoção mais presente.

No caso do autocontrole dos sentimentos e das emoções houve magnitudes nulas com as emoções alegria e coragem, assim como magnitudes baixas com o medo em ambos os sexos e com a tristeza no caso dos meninos. Por sua vez, as meninas apresentaram um coeficiente de correlação considerado moderado entre a tristeza e esse tipo de autocontrole, com uma comunalidade entre os construtos de aproximadamente $25 \%$. Com base nisso, concluiu-se que haveria evidência de validade concorrente entre as emoções tristeza e medo com o fator referente a sentimentos e emoções das escalas de autocontrole, pois deve ser destacado que os coeficientes apresentados entre este tipo de autocontrole e as emoções foi bastante superior ao autocontrole das normas e condutas sociais.

Com base nesses resultados, pode-se acrescentar que este estudo apresentou evidências de validade para as Escalas Feminina e Masculina de Autocontrole (Martinelli, \& Sisto, 2007) e para a Escala de Autopercepção de Emoções (Sisto \& Bazi 1999). No entanto, sugerem-se novos estudos com o objetivo de aprofundar os achados desta pesquisa.

\section{Referências}

Agostin, T. M., \& Bain, S. K. (1997). Predicting early school success with developmental and social skills screeners. Psychology in the Schools, 34(3), 219-228.

Dolores Avia, M. (1995). Personas felices: las emociones positivas. Em Dolores Avia, M. \& Sánchez Bernardos, M. L. Personalidad: Aspectos cognitivos y sociales. Madrid: Pirámide.

Dutrow, A. M. \& Houston, C. A. (1981). An analysis of the ralationships of academic success and selected achievement / attitude tests. In Anais, Annual Meeting of the Southeastern Association for Community College Research, 1981. Orlando. Orlando: Association for Community College Research.

Frijda, N. H. (1995). Las leis de la emoción. Em Dolores Avia, M. \& Sánchez Bernardos, M. L. Personalidad: Aspectos cognitivos y sociales. Madrid: Pirámide.

Harris, G. \& Johnson, S. B. (1980). Comparison of individualized covert modeling, self-control desensitization, and study skills training for alleviation of test anxiety. Journal of Consulting and Clinical Psychology, 48(2), 186-194.

Harris, K. R., \& Graham, S. (1985). Improving learning disabled students' composition skills: self-control strategy training. Learning Disability Quarterly, 8(1), 27-36. 
Herman, S. M. (1971). Toward a more authentic manager. Training and Development Journal, 25(10), 8-10.

Kearney, D. L. (1966). Selected non-intellectual factors as predictors of academic success in junior college intellectually capable students. Tese de doutorado, University of Southern California. Los Angeles.

Konopkin, O. A., Stepanskii, V. I., \& Kondrat'eva, I. I. (1973). Methodological characteristics of the organization of reaction time experiments. Voprosy Psikhologii, 19(4), 159-164.

Marino Junior, R. (1975). Fisiologia das emoções: Introdução à neurologia do comportamento, anatomia e funções do sistema límbico. São Paulo: Sarvier.

Martinelli, S. C. \& Sisto, F. F. (2007). Escalas Feminina e Masculina de Autocontrole. São Paulo: Vetor.

May, R. (1975). The courage to create. Norton, NY: W.W.

Misrahi, R. (2001). A felicidade: Ensaio sobre a alegria. Rio de Janeiro: DIFEL.

Saltz, E., \& Meade, E. (1973). Verbal control of impulsive behavior in the classroom. Detroit: Wayne State University.

Sappington, A. A., Fritschi, O., Sandefer, D., \& Tauxe, M. (1980). Self-directed study skill programs for students on probation. Journal of Counseling Psychology, 27(6), 616-619.

Sisto, F. F., \& Bazi, G. A. P. (1999). Escala de autopercepção de emoções. Faculdade de Educação, Unicamp (mimeo).

Sisto, F. F. (2004). Escala de traços de personalidade para criança. São Paulo: Vetor.

Sisto, F. F., Bartholomeu, D., Rueda, F. J. M., \& Fernandes, D. C. (2004). Autoconceito e Emoções. In Machado, C., Almeida, L. S., Gonçalves, M., \& Ramalho, V. (Org.). Avaliação psicológica: Formas e contextos (pp. 67-74). Braga: Psiquilíbrios.

Sisto, F. F., \& Rueda, F. J. M. (2006). Escalas de avaliação da percepção de autocontrole e traços de personalidade: Evidências de validade. São Paulo: Manuscrito submetido à publicação.
Stevenson, H. C., \& Fantuzzo, J. W. (1986). The generality and social validity of a competencybased self-control training intervention for underachieving students. Journal of Applied Behavior Analysis, 19(3), 269-276.

Verztman, J. S. (1995). Tristeza e depressão: Pensando nos problemas da vida. Rio de Janeiro: Vozes.

Whiteside, R. (1966). A study of methods of controlling impulses. Abilene: Abilene Christian College.

Wolfe, R. N., \& Johnson, S. D. (1995). Personality as a predictor of college performance. Educational and Psychological Measurement, 55(2), 177-185.
Recebido em: 29/05/2007

Received in: 05/29/2007

Aprovado em: 24/10/2007

Approved in: 10/24/2007 